\title{
Pulsation Investigation of V647 Tau - Preliminary Results of STEPHI Campaign in 1997
}

\author{
Y. Y. Liu
}

Beijing Astronomical Observatory, 100012, China

E. Michel, C. Barban

Observatoire de Meudon, DASGAL, F-92195, France

R. Garrido

Instituto de Astrofisica de Andalucía, E-18080 Granada, Spain

M. Alvarez

Instituto de Astronomía-UNAM, Ensenada, BC, México

M. M. Hernández, J. A. Belmonte

Instituto de Astrofisica de Canarias, E-38200 La Laguna, Spain

\begin{abstract}
V647 Tan was observed during a 1997 STEPHI campaign; $228 \mathrm{hr}$ of observations in $v$ were obtained, together with another $50 \mathrm{hr}$ of simultaneous data in $y$. The data were analyzed and main results are discussed in the text.
\end{abstract}

\section{Observation}

From 1997 November 19 to December 13, high-speed, high-accuracy photometry of the $\delta$ Scuti star V647 Tau in the Pleiades cluster was carried out by STEPHI (STEllar PHotometry International). Chevreton four-channel photometers were used in San Pedro Mártir, Observatoire de Haute-Provence, and Observatorio del Teide with a $v$ filter. In Xinglong, simultaneous $v$ and $y$ were measured using a six-channel photometer. The long observational time base $(228 \mathrm{hr})$ gives rise to a frequency resolution $\Delta \nu=0.5 \mu \mathrm{Hz}$, with an effective time coverage of $38 \%$.

\section{Analysis}

Each night data of every observatory was normalized to zero point and merged into one time series after correcting the effects of instrumental drift, atmospheric extinction and fluctuation in transparency. Fourier transforms were performed using code MFA (Hao 1991). Five frequencies listed in Table 1 were unambiguously determined. To confirm the result, the above procedures were also 
repeated on data from the two comparison stars. None of the five frequencies is found in the power spectra of the comparison stars. The five-frequency solution was then applied to the $v$ and $y$ datasets of Xinglong in an effort to obtain the corresponding amplitude and phase by means of least squares. The amplitude ratio and phase shift between the two filters were derived. Theoretical values were also calculated according to Garrido, García-Lobo, \& Rodríguez (1990).

\section{Discussion}

An earlier observation of V647 Tau gave a frequency $f=20.41 \mathrm{~d}^{-1}$ and light variation $\Delta V=0.01$ (Breger 1972). This is, in fact, none other than $\nu_{2}$ in Table 1, with a decreased amplitude. The global parameters are derived from photometric calibration according to Domingo \& Figueras (1999). They are $T_{\text {eff }}=7881 \pm 270 \mathrm{~K}, \log g=4.26 \pm 0.18$, and $M_{V}=2.39 \pm 0.22$. The Q-values are then calculated and listed in Table 1 . Note that the period ratio $P_{3} / P_{2} \approx 0.75$. $\mathrm{Q}_{2}$ and $\mathrm{Q}_{3}$ are typical of radial pulsation, satisfying the relation between radial fundamental and first overtone. A model calculation also shows that $\nu_{2}$ has a large positive phase shift, while the others are negative. Presently, we can only say that $\nu_{2}$ and $\nu_{4}$ are radial and $\ell=2$ nonradial modes respectively; the other three modes cannot be determined, considering their error bars.

Table 1. The results of a period analysis for V647 Tau.

\begin{tabular}{rrrrrrc}
\hline No. & $\begin{array}{r}\text { Frequency } \\
\mathrm{d}^{-1}\end{array}$ & $\begin{array}{r}\text { Amp. } \\
\text { mmag }\end{array}$ & $\begin{array}{r}\text { Phase } \\
(\times 2 \pi \text { rad })\end{array}$ & $\mathrm{A}(v) / \mathrm{A}(y)$ & $\begin{array}{r}\phi(v)-\phi(y) \\
\text { degree }\end{array}$ & $\begin{array}{c}\mathrm{Q} \\
\left(\times 10^{-2}\right)\end{array}$ \\
\hline 1 & 32.3494 & 3.12 & 0.279 & 1.443 & -2.270 & 1.95 \\
& \pm 4 & \pm 5 & \pm 6 & \pm 0.040 & \pm 1.634 & \\
2 & 20.4375 & 2.13 & 0.897 & 1.196 & 3.930 & 3.09 \\
& \pm 6 & \pm 5 & \pm 8 & \pm 0.049 & \pm 2.342 & \\
3 & 27.2616 & 1.93 & 0.926 & 1.428 & -6.217 & 2.31 \\
& \pm 7 & \pm 6 & \pm 9 & \pm 0.076 & \pm 3.066 & \\
4 & 38.3704 & 1.43 & 0.599 & 1.308 & -15.928 & 1.64 \\
& \pm 9 & \pm 5 & \pm 12 & \pm 0.076 & \pm 3.341 & \\
5 & 26.3206 & 1.30 & 0.976 & 1.433 & -9.319 & 2.40 \\
& \pm 10 & \pm 6 & \pm 14 & \pm 0.103 & \pm 4.069 & \\
\hline
\end{tabular}

\section{References}

Breger, M. 1972, ApJ, 176, 367

Domingo, A. \& Figueras, F. 1999, A\&A, 343, 446

Garrido, R., García-Lobo, E., \& Rodríguez, E. 1990, A\&A, 234, 262

Hao, J. 1991, Pub. of the Beijing Astro. Obs., 18, 35 